\title{
Application of Optimal Homotopy Asymptotic Method to Burger Equations
}

\author{
R. Nawaz, H. Ullah, S. Islam, and M. Idrees \\ Department of Mathematics, Abdul Wali Khan University, Mardan 23200, Pakistan \\ Correspondence should be addressed to H. Ullah; hakeemullahl@gmail.com
}

Received 8 April 2013; Accepted 10 June 2013

Academic Editor: Anjan Biswas

Copyright ( 2013 R. Nawaz et al. This is an open access article distributed under the Creative Commons Attribution License, which permits unrestricted use, distribution, and reproduction in any medium, provided the original work is properly cited.

We apply optimal homotopy asymptotic method (OHAM) for finding approximate solutions of the Burger's-Huxley and Burger'sFisher equations. The results obtained by proposed method are compared to those of Adomian decomposition method (ADM) (Ismail et al., (2004)). As a result it is concluded that the method is explicit, effective, and simple to use.

\section{Introduction}

Nonlinear phenomena play a vital role in applied mathematics, physics, and engineering sciences. The Burger's equation models efficiently certain problems of a fluid flow nature, in which either shocks or viscous dissipation is a significant factor. It can be used as a model for any nonlinear wave propagation problem subject to dissipation [1]. The first steadystate solutions of Burger equation were given by Young et al. [2] However, the equation gets its name from the extensive research of Burger's [3]. The generalized Burger's-Huxley introduced by Satsuma shows a prototype model for describing the communication among reaction mechanisms, convection effects, and diffusion transports [4]. Burger-Fisher equation has significant applications in various fields of applied mathematics and has physical applications such as gas dynamic, traffic flow, convection effect, and diffusion transport [5-12]. Marinca and Herişanu et al. introduced a new semianalytic method OHAM for approximate solution of nonlinear problems of thin film flow of a fourth-grade fluid down a vertical cylinder. In progression of papers Marinca and Herişanu et al. have applied this method for the solution of nonlinear equations arising in the steady state flow of a fourth-grade fluid past a porous plate and for the solution of nonlinear equations arising in heat transfer [13-15]. The method has been applied by a number of researchers for solution of ordinary and partial differential equations [1621]. The motivation of this paper is to show the effectiveness of OHAM for the solution of Burger's-Huxley and Burger'sFisher equations. We consider Burger's-Huxley equation of the form

$$
\begin{aligned}
& \frac{\partial u(x, t)}{\partial t}+\alpha u^{\delta}(x, t) \frac{\partial u(x, t)}{\partial x}-\frac{\partial^{2} u(x, t)}{\partial x^{2}} \\
&-\beta u(x, t)\left(1-u^{\delta}(x, t)\right)\left(u^{\delta}(x, t)-\gamma\right)=0, \\
& \forall 0 \leq x \leq 1, t \geq 0
\end{aligned}
$$

and Burger's-Fisher equation of the form

$$
\begin{array}{r}
\frac{\partial u(x, t)}{\partial t}+\alpha u^{\delta}(x, t) \frac{\partial u(x, t)}{\partial x}-\frac{\partial^{2} u(x, t)}{\partial x^{2}} \\
-\beta u(x, t)\left(1-u^{\delta}(x, t)\right)=0, \\
\forall 0 \leq x \leq 1, t \geq 0,
\end{array}
$$

where $\alpha, \beta, \gamma$, and $\delta$ are parameters and $\beta \geq 0, \delta \geq 0, \gamma \in$ $(0,1)$.

The present paper is divided into three sections. In Section 2 fundamental mathematical theory of OHAM is presented. In Section 3 comparisons are made between the results of the proposed method and HAM for Burger'sHuxley. In Section 4 solution of Burger's-Fisher equation is presented, and absolute error of approximate solution of proposed method is compared with approximate solution of HAM. In all cases the proposed method yields better results than those of ADM. 


\section{Fundamental Theory of OHAM}

Here we start by describing the basic idea of OHAM. Consider the partial differential equation of the form:

$$
\begin{gathered}
\mathscr{L}(u(x, t))+\mathscr{N}(u(x, t))+\mathscr{q}(x, t)=0, \quad x \in \Omega, \\
\mathscr{B}\left(u, \frac{\partial u}{\partial t}\right)=0,
\end{gathered}
$$

where $\mathscr{L}$ is a linear operator and $\mathscr{N}$ is nonlinear operator. $\mathscr{B}$ is boundary operator, $u(x, t)$ is an unknown function, and $x$ and $t$ denote spatial and time variables, respectively; $\Omega$ is the problem domain and $g(x, t)$ is a known function.

According to the basic idea of OHAM, one can construct the optimal homotopy $\psi(x, t ; q): \Omega \times[0,1] \rightarrow R$ which satisfies

$$
\begin{aligned}
(1-q) & \{\mathscr{L}(\psi(x, t ; q))+g(x, t)\} \\
= & \mathscr{H}(q)\{\mathscr{L}(\psi(x, t ; q))+\mathscr{N}(\psi(x, t ; q))+\mathscr{g}(x, t)\},
\end{aligned}
$$

where $q \in[0,1]$ is an embedding parameter, $H(q)$ is a nonzero auxiliary function for $q \neq 0, H(0)=0$. Equation (3) is called optimal homotopy equation. Clearly, we have

$$
\begin{aligned}
q & =0 \Longrightarrow \mathscr{H}(\psi(x, t ; 0), 0)=\mathscr{L}(\psi(x, t ; 0))+\mathscr{g}(x, t)=0 \\
q & =1 \Longrightarrow \mathscr{H}(\psi(x, t ; 1), 1) \\
& =\mathscr{H}(1)\{\mathscr{L}(\psi(x, t ; q))+\mathscr{N}(\psi(x, t ; q))+\mathscr{L}(x, t)\}=0 .
\end{aligned}
$$

Clearly, when $q=0$ and $q=1$, it holds that $\psi(x, t ; 0)=$ $u_{0}(x, t)$ and $\psi(x, t ; 1)=u(x, t)$, respectively. Thus, as $q$ varies from 0 to 1 , the solution $\psi(x, t ; q)$ approaches from $u_{0}(x, t)$ to $u(x, t)$, where $u_{0}(x, t)$ is obtained from (3) for $q=0$ :

$$
\mathscr{L}\left(u_{0}(x, t)\right)+g(x, t)=0, \quad \mathscr{B}\left(u_{0}, \frac{\partial u_{0}}{\partial t}\right)=0 .
$$

Next, we choose auxiliary function $H(q)$ in the form

$$
\mathscr{H}(q)=q C_{1}+q^{2} C_{2}+\cdots .
$$

Here $C_{1}, C_{2}, \ldots$ are constants to be determined later.

To get an approximate solution, we expand $\psi\left(x, t ; q, C_{i}\right)$ in Taylor's series about $q$ in the following manner:

$$
\psi\left(x, t ; q, C_{i}\right)=u_{0}(x, t)+\sum_{k=1}^{\infty} u_{k}\left(x, t ; C_{i}\right) q^{k}, \quad i=1,2, \ldots
$$

Substituting (10) into (4) and equating the coefficient of like powers of $q$, we obtain Zeroth-order problem, given by (6), the first- and second-order problems are given by (11)-(12), respectively, and the general governing equations for $u_{k}(x, t)$ are given by (13):

$$
\begin{aligned}
& \mathscr{L}\left(u_{1}(x, t)\right)=C_{1} \mathcal{N}_{0}\left(u_{0}(x, t)\right), \quad \mathscr{B}\left(u_{1}, \frac{\partial u_{1}}{\partial t}\right)=0 \\
& \mathscr{L}\left(u_{2}(x, t)\right)-\mathscr{L}\left(u_{1}(x, t)\right) \\
& =C_{2} \mathcal{N}_{0}\left(u_{0}(x, t)\right) \\
& +C_{1}\left[\mathscr{L}\left(u_{1}(x, t)\right)+\mathscr{N}_{1}\left(u_{0}(x, t), u_{1}(x, t)\right)\right] \\
& \mathscr{B}\left(u_{2}, \frac{\partial u_{2}}{\partial t}\right)=0 \\
& \mathscr{L}\left(u_{k}(x, t)\right)-\mathscr{L}\left(u_{k-1}(x, t)\right) \\
& =C_{k} \mathscr{N}_{0}\left(u_{0}(x, t)\right) \\
& +\sum_{i=1}^{k-1} C_{i}\left[\mathscr{L}\left(u_{k-i}(x, t)\right)+\mathcal{N}_{k-i}\right. \\
& \left.\quad \times\left(u_{0}(x, t), u_{1}(x, t), \ldots, u_{k-i}(x, t)\right)\right] \\
& \mathscr{B}\left(u_{k}, \frac{\partial u_{k}}{\partial t}\right)=0, \quad k=2,3, \ldots
\end{aligned}
$$

where $\mathcal{N}_{k-i}\left(u_{0}(x, t), u_{1}(x, t), \ldots, u_{k-i}(x, t)\right)$ is the coefficient of $q^{k-i}$ in the expansion of $\mathcal{N}(\psi(x, t ; q))$ about the embedding parameter $q$ :

$$
\begin{aligned}
\mathcal{N}\left(\psi\left(x, t ; q, C_{i}\right)\right)= & \mathcal{N}_{0}\left(u_{0}(x, t)\right) \\
& +\sum_{k \geq 1} \mathcal{N}_{k}\left(u_{0}, u_{1}, u_{2}, \ldots, u_{k}\right) q^{k} .
\end{aligned}
$$

Here $u_{k}$ for $k \geq 0$ are set of linear equations with the linear boundary conditions, which can be easily solved.

The convergence of the series in (10) depends upon the auxiliary constants $C_{1}, C_{2}, \ldots$ If it is convergent at $q=1$, one has:

$$
\widetilde{u}\left(x, t ; C_{i}\right)=u_{0}(x, t)+\sum_{k \geq 1} u_{k}\left(x, t ; C_{i}\right) .
$$

Substituting (15) into (1) results in the following expression for residual:

$$
\mathscr{R}\left(x, t ; C_{i}\right)=\mathscr{L}\left(\widetilde{u}\left(x, t ; C_{i}\right)\right)+\mathscr{L}(x, t)+\mathscr{N}\left(\widetilde{u}\left(x, t ; C_{i}\right)\right) .
$$

If $R\left(x, t ; C_{i}\right)=0$, then $\widetilde{u}\left(x, t ; C_{i}\right)$ will be the exact solution.

For computing the auxiliary constants, $C_{i}, i=1,2, \ldots, m$, there are many methods like Galerkin's Method, Ritz Method, Least Squares Method, and Collocation Method to find the optimal values of $C_{i}, i=1,2,3, \ldots$, One can apply the Method of Least Squares as

$$
\mathscr{I}\left(C_{i}\right)=\int_{0}^{t} \int_{\Omega} \mathscr{R}^{2}\left(x, t, C_{i}\right) d x d t
$$


where $R$ is the residual, $R\left(x, t ; C_{i}\right)=L\left(\widetilde{u}\left(x, t ; C_{i}\right)\right)+g(x, t)+$ $N\left(\widetilde{u}\left(x, t ; C_{i}\right)\right)$, and

$$
\frac{\partial \mathscr{I}}{\partial C_{1}}=\frac{\partial \mathscr{I}}{\partial C_{2}}=\cdots=\frac{\partial \mathscr{I}}{\partial C_{m}}=0 .
$$

The constants $C_{i}$ can also be determined by another method as

$$
\begin{array}{r}
\mathscr{R}\left(h_{1} ; C_{i}\right)=\mathscr{R}\left(h_{2} ; C_{i}\right)=\cdots=\mathscr{R}\left(h_{m} ; C_{i}\right)=0, \\
i=1,2, \ldots, m,
\end{array}
$$

at any time $t$, where $h_{i} \in \Omega$. The convergence depends upon constants $C_{1}, C_{2}, \ldots$, can be optimally identified and minimized by (18).

\section{Application of OHAM}

In this section we apply OHAM for the two problems: the first is the Burger's-Huxley equation (1) and the second is the Burger's-Fisher equation (2).

3.1. Application of OHAM for Burger's-Huxley Equation. Let us consider Burger's-Huxley equation of form (1):

$$
\begin{array}{r}
\frac{\partial u(x, t)}{\partial t}+\alpha u^{\delta}(x, t) \frac{\partial u(x, t)}{\partial x}-\frac{\partial^{2} u(x, t)}{\partial x^{2}} \\
-\beta u(x, t)\left(1-u^{\delta}(x, t)\right)\left(u^{\delta}(x, t)-\gamma\right)=0, \\
\forall 0 \leq x \leq 1, t \geq 0,
\end{array}
$$

Subject to constant initial condition

$$
u(x, 0)=\left(0.5 \gamma+0.5 \gamma \tanh \left(A_{1} x\right)\right)^{1 / \delta} .
$$

The exact solution of (26) with given condition is given by

$$
u(x, 0)=\left(0.5 \gamma+0.5 \gamma \tanh \left(A_{1}\left(x-A_{2} t\right)\right)\right)^{1 / \delta},
$$

where

$$
\begin{gathered}
A_{1}=\frac{-\alpha \delta+\delta \sqrt{\alpha^{2}+4 \beta(1+\delta)}}{4(1+\delta)} \gamma, \\
A_{2}=\frac{\alpha \gamma}{(1+\delta)}-\frac{(1+\delta-\gamma)\left(-\alpha+\sqrt{\alpha^{2}+4 \beta(1+\delta)}\right)}{2(1+\delta)} .
\end{gathered}
$$

For computational work, we have taken $\alpha=1, \beta=1, \delta=1$, and $\gamma=0.001$ for various values of $x$ and $t$.

Following the basic idea of OHAM presented in preceding section we start with

Zeroth-Order Problem

$$
\begin{gathered}
\frac{\partial u_{0}(x, t)}{\partial t}=0 \\
u_{0}(x, 0)=(0.0005+0.0005 \tanh (0.00025 x)) .
\end{gathered}
$$

Its solution is

$$
u_{0}(x, t)=(0.0005+0.0005 \tanh (0.00025 x)) .
$$

First-Order Problem

$$
\begin{gathered}
\frac{\partial u_{1}(x, t)}{\partial t}-\left(1+C_{1}\right) \frac{\partial u_{0}(x, t)}{\partial t}-C_{1} u_{0}(x, t) \frac{\partial u_{0}(x, t)}{\partial x} \\
+C_{1} \frac{\partial^{2} u_{0}(x, t)}{\partial x^{2}}-0.001 C_{1} u_{0}(x, t)+0.001 C_{1} u_{0}^{2}(x, t) \\
-C_{1} u_{0}^{3}(x, t)=0, \\
u_{1}(x, 0)=0 .
\end{gathered}
$$

Its solution is

$$
\begin{aligned}
u_{1}(x, t) & \\
=-t & \left(-2.4987500000000003 \times 10^{-7} C_{1}\right. \\
& -6.25 \times 10^{-11} C_{1} \operatorname{sech}^{2}(0.00025 x) \\
& +1.2499999999999317 \\
& \times 10^{-10} C_{1} \tanh (0.00025 x) \\
& -1.25 \times 10^{-10} C_{1} \operatorname{sech}^{2}(0.00025 x) \\
& \times \tanh (0.00025 x) \\
& +2.49875 \times 10^{-7} C_{1} \tanh ^{2}(0.00025 x) \\
& \left.-1.25 \times 10^{-10} C_{1} \tanh ^{3}(0.00025 x)\right) .
\end{aligned}
$$

Second-Order Problem

$$
\begin{gathered}
\frac{\partial u_{2}(x, t)}{\partial t}-\left(1+C_{1}\right) \frac{\partial u_{1}(x, t)}{\partial t}-C_{2} u_{0}(x, t) \frac{\partial u_{0}(x, t)}{\partial x} \\
+C_{2} \frac{\partial^{2} u_{0}(x, t)}{\partial x^{2}}-C_{2} \frac{\partial u_{0}(x, t)}{\partial t}-C_{1} u_{1}(x, t) \frac{\partial u_{0}(x, t)}{\partial x} \\
-C_{1} u_{0}(x, t) \frac{\partial u_{1}(x, t)}{\partial x}+C_{1} \frac{\partial^{2} u_{1}(x, t)}{\partial x^{2}}-0.001 C_{2} u_{0}(x, t) \\
+1.001 C_{2} u_{0}^{2}(x, t)-C_{2} u_{0}^{3}(x, t)-0.001 C_{1} u_{1}(x, t) \\
+2.002 C_{1} u_{0}(x, t) u_{1}(x, t)-3 C_{1} u_{0}^{2}(x, t) u_{1}(x, t)=0, \\
u_{2}(x, 0)=0 .
\end{gathered}
$$

Its solution is

$$
\begin{aligned}
u_{2}(x, t, & \left.C_{1}\right) \\
= & -t-2.4987500000000003 \times 10^{-7} C_{1}-6.25 \\
& \times 10^{-11} C_{1} \operatorname{sech}^{2}(0.00025 x) \\
& +1.2499999999999317 \times 10^{-10} C_{1}
\end{aligned}
$$




$$
\begin{aligned}
& \times \tanh (0.00025 x)-1.25 \times 10^{-10} C_{1} \\
& \times \operatorname{sech}^{2}(0.00025 x) \tanh (0.00025 x) \\
& +2.49875 \times 10^{-7} C_{1} \tanh ^{2}(0.00025 x) \\
& -1.25 \times 10^{-10} C_{1} \tanh ^{3}(0.00025 x)
\end{aligned}
$$

\section{Third-Order Problem}

$$
\begin{aligned}
& \frac{\partial u_{3}(x, t)}{\partial t}-\left(1+C_{1}\right) \frac{\partial u_{2}(x, t)}{\partial t}-C_{3} u_{0}(x, t) \frac{\partial u_{0}(x, t)}{\partial x} \\
& +C_{3} \frac{\partial^{2} u_{0}(x, t)}{\partial x^{2}}-C_{3} \frac{\partial u_{0}(x, t)}{\partial t}-C_{2} u_{1}(x, t) \frac{\partial u_{0}(x, t)}{\partial x} \\
& -C_{2} u_{0}(x, t) \frac{\partial u_{1}(x, t)}{\partial x}+C_{2} \frac{\partial^{2} u_{1}(x, t)}{\partial x^{2}}-C_{2} \frac{\partial u_{1}(x, t)}{\partial t} \\
& -C_{1} u_{2}(x, t) \frac{\partial u_{0}(x, t)}{\partial x}-C_{1} u_{1}(x, t) \frac{\partial u_{1}(x, t)}{\partial x} \\
& +C_{1} \frac{\partial^{2} u_{2}(x, t)}{\partial x^{2}}-C_{1} u_{0}(x, t) \frac{\partial u_{2}(x, t)}{\partial x} \\
& -0.001 C_{3} u_{0}(x, t)+1.001 C_{3} u_{0}^{2}(x, t) \\
& -C_{3} u_{0}^{3}(x, t)-0.001 C_{2} u_{1}(x, t) \\
& -3 C_{2} u_{0}^{2}(x, t) u_{1}(x, t)+1.001 C_{1} u_{1}^{2}(x, t) \\
& -3 C_{1} u_{0}(x, t) u_{1}^{2}(x, t)-0.001 C_{1} u_{2}(x, t) \\
& +2.002 C_{1} u_{0}(x, t) u_{2}(x, t)-3 C_{1} u_{0}^{2}(x, t) u_{2}(x, t) \\
& +2.002 C_{2} u_{0}(x, t) u_{1}(x, t)=0 \\
& u_{3}(x, 0)=0 \\
& +2
\end{aligned}
$$

Its solution is

$$
\begin{aligned}
& u_{3}\left(x, t, C_{1}, C_{2}, C_{3}\right) \\
& =\left(\frac{1}{\left(1+e^{0.5 x}\right)^{2}} t \operatorname{sech}^{2}(0.25 x)\right. \\
& \times\left(-0.0625 C_{2}-0.0625 C_{3}\right. \\
& +C_{1}\left(-0.0625+C_{1}(-0.125-0.015625 t)\right. \\
& +C_{2}(-0.125-0.015625 t) \\
& \quad-0.000651042 C_{1}^{2}(5.0718+t) \\
& \quad \times(18.9282+t)) \\
& +\left(\left(288 C_{2}+288 C_{3}+C_{1}\right.\right. \\
& \quad \times\left(288+576 C_{2}+C_{1}(576-24 t)\right.
\end{aligned}
$$$$
-24 C_{2} t-3 C_{1}^{2}(-6.58301+t)
$$$$
\times(14.583+t) \cosh (0.25 x)
$$$$
+\left(-96 C_{2}-96 C_{3}+C_{1}\right.
$$$$
\times\left(-96-192 C_{2}+C_{1}(-192-24 t)\right.
$$$$
-24 C_{2} t+5 C_{1}^{2}(-7.396+t)
$$$$
\times(2.596+t))) \sinh (0.25 x))
$$$$
\left.\left.-24 C_{2} t+5 C_{1}^{2}(-7.396+t)(2.596+t)\right)\right)
$$

$\times \sinh (0.25 x)$

$\times(-0.000651042 \cosh (0.75 x)$

$$
-0.000651042 \sinh (0.75 x))) \text { ). }
$$

Adding (25), (27), (29), and (31) we obtain

$$
\begin{aligned}
\tilde{u}\left(x, t, C_{1}, C_{2}\right)= & u_{0}(x, t)+u_{1}\left(x, t, C_{1}\right) \\
& +u_{2}\left(x, t, C_{1}, C_{2}\right)+u_{3}\left(x, t, C_{1}, C_{2}, C_{3}\right) .
\end{aligned}
$$

For the calculations of the constants $C_{1}, C_{2}$, and $C_{3}$ using the collocation method, we have computed that

$$
\begin{aligned}
& C_{1}=-1.0000010231545267, \\
& C_{2}=-9.98159444155818 \times 10^{-7}, \\
& C_{3}=-2.041939789528322 \times 10^{-12} .
\end{aligned}
$$

Putting the values of these constants into (32) the third order approximate solution using OHAM is

$$
\begin{aligned}
& u_{3}(x, t) \\
& =0.5-5.2607845247854 \times 10^{-6} t \operatorname{sech}^{2}(0.25 x) \\
& +\frac{1}{\left(1+e^{0.5 x}\right)^{2}} \\
& \times\left(6.360090415451543 \times 10^{-9}\right. \\
& \quad+\left(1.3153067008227217 \times 10^{-6}\right. \\
& \quad+0.0006512058813062292 t) t \\
& \quad+\left(1.2720180853076355 \times 10^{-8}\right. \\
& \left.\quad-0.002604823525224917 t^{2}\right) \cosh (0.5 x)
\end{aligned}
$$


TABLE 1: Comparison of absolute errors of OHAM and ADM [5] for $\alpha=1, \beta=1, \delta=1$, and $\gamma=0.001$.

\begin{tabular}{lcccccc}
\hline$t$ & ADM & OHAM & ADM & OHAM & ADM & OHAM \\
& $x=0.1$ & $x=0.1$ & $x=0.5$ & $x=0.5$ & $x=0.9$ \\
\hline 0.05 & $1.93715 \times 10^{-7}$ & $1.87406 \times 10^{-8}$ & $1.9373 \times 10^{-7}$ & $1.87406 \times 10^{-8}$ & $1.93745 \times 10^{-7}$ & $1.87406 \times 10^{-8}$ \\
0.1 & $3.87434 \times 10^{-7}$ & $3.74812 \times 10^{-8}$ & $3.87464 \times 10^{-7}$ & $3.74812 \times 10^{-8}$ & $3.87494 \times 10^{-7}$ & $3.74812 \times 10^{-8}$ \\
1 & $3.87501 \times 10^{-6}$ & $3.74812 \times 10^{-7}$ & $3.87531 \times 10^{-6}$ & $3.74812 \times 10^{-7}$ & $3.87561 \times 10^{-6}$ & $3.74812 \times 10^{-7}$ \\
\hline
\end{tabular}

TABLE 2: Comparison of absolute errors obtained by OHAM and ADM [5] for $\alpha=0, \beta=1, \delta=1$, and $\gamma=0.001$.

\begin{tabular}{lcccccc}
\hline$t$ & ADM & OHAM & ADM & OHAM & ADM & OHAM \\
$x=0.9$ & for $x=0.5$ & $x=0.9$ \\
\hline 0.05 & $1.93715 \times 10^{-7}$ & $2.49875 \times 10^{-8}$ & $1.9373 \times 10^{-7}$ & $2.49875 \times 10^{-8}$ & $1.93745 \times 10^{-7}$ & $2.49875 \times 10^{-8}$ \\
0.1 & $3.87434 \times 10^{-7}$ & $4.9975 \times 10^{-8}$ & $3.87464 \times 10^{-7}$ & $4.9975 \times 10^{-8}$ & $3.87494 \times 10^{-7}$ & $4.9975 \times 10^{-8}$ \\
1 & $3.87501 \times 10^{-6}$ & $4.9975 \times 10^{-7}$ & $3.87531 \times 10^{-6}$ & $4.9975 \times 10^{-7}$ & $3.87561 \times 10^{-6}$ & $4.9975 \times 10^{-7}$ \\
\hline
\end{tabular}

TABLE 3: Comparison of absolute errors obtained by OHAM and ADM [5] for $\alpha=0, \beta=1, \delta=2$, and $\gamma=0.001$.

\begin{tabular}{lcc}
\hline$t$ & Error ADM & Error OHAM \\
\hline 0.05 & $5.58836 \times 10^{-7}$ & $2.7938 \times 10^{-7}$ \\
0.1 & $1.11766 \times 10^{-6}$ & $5.58771 \times 10^{-7}$ \\
1 & $1.00741 \times 10^{-5}$ & $5.5896 \times 10^{-6}$ \\
\hline
\end{tabular}

$$
\begin{aligned}
& +\left(6.36009042653818 \times 10^{-9}\right. \\
& +\left(-1.3153067008227217 \times 10^{-6}\right. \\
& \quad+0.0006512058813062292 t) t) \cosh (x) \\
& +\left(1.2720180853076355 \times 10^{-8}\right. \\
& \left.\quad-0.002604823525224917 t^{2}\right) \sinh (0.5 x) \\
& +\left(6.36009042653818 \times 10^{-9}\right. \\
& +\left(-1.3153067008227217 \times 10^{-9}\right. \\
& \quad+0.0006512058813062292 t) t) \sinh (x) \\
& -0.5 \tanh ^{-9}(0.25 x)+0.007813813661895406 t^{2} \\
& \times \operatorname{sech}(0.25 x) \tanh (0.25 x)+\operatorname{sech}^{2}(0.25 x) \\
& \times(0.06250525442670962 t)) .
\end{aligned}
$$

Table 1 shows a comparison between OHAM solution and ADM solution for $\alpha=1$ and $\gamma=0.001$. For $\alpha=0$ (1) is reduced to the generalized Huxley equation which describes nerve pulse propagation in nerve fibers and wall motion in liquid crystals [22]. Tables 2 and 3 show a comparison between ADM solution and OHAM solution for $\alpha=0$ and $\beta=1$ respectively. Table 4 shows absolute errors of OHAM solution for larger domain for $\alpha=0,1, \beta=1$, and $\delta=1,2$ respectively.
3.2. Application of OHAM for Burger's-Fisher Equation. Consider the Burger's-Fisher equation of form (2):

$$
\begin{aligned}
& \frac{\partial u(x, t)}{\partial t}+\alpha u^{\delta}(x, t) \frac{\partial u(x, t)}{\partial x}-\frac{\partial^{2} u(x, t)}{\partial x^{2}} \\
&-\beta u(x, t)\left(1-u^{\delta}(x, t)\right)=0, \\
& \forall 0 \leq x \leq 1, t \geq 0,
\end{aligned}
$$

subject to constant initial condition

$$
u(x, 0)=\left(0.5+0.5 \tanh \left(\frac{-\alpha \delta}{2(\delta+1)} x\right)\right)^{1 / \delta}
$$

with exact solution given by

$$
\begin{aligned}
u(x, t)= & 0.5 \\
\times & +0.5 \tanh \\
& =\left(\frac{-\alpha \delta}{2(\delta+1)}\right. \\
& \left.\times\left(x-\left(\frac{\alpha}{(\delta+1)}+\frac{\beta(\delta+1)}{\alpha}\right) t\right)\right)^{1 / \delta} .
\end{aligned}
$$

For computational work, we have taken $\alpha=0.001, \beta=0.001$, and $\delta=1$ for various values of $x$ and $t$.

\section{Zeroth-Order Problem}

$$
\begin{gathered}
\frac{\partial u_{0}(x, t)}{\partial t}=0 \\
u_{0}(x, 0)=\left(0.5+0.5 \tanh \left(\frac{-0.001}{4} x\right)\right)
\end{gathered}
$$

Its solution is

$$
u_{0}(x, t)=\left(0.5-0.5 \tanh \left(\frac{0.001}{4} x\right)\right)
$$


TABLE 4: Absolute errors of OHAM for $\alpha=0,1, \beta=1, \delta=1,2$, and $x=2$.

\begin{tabular}{lccc}
\hline$t$ & $\alpha=1, \beta=1, \delta=1$, and $\gamma=0.001$ & $\alpha=0, \beta=1, \delta=1$, and $\gamma=0.001$ & $\alpha=0, \beta=1, \delta=2$, and $\gamma=0.001$ \\
\hline 0.1 & $3.74812 \times 10^{-8}$ & $2.49875 \times 10^{-8}$ & $2.23403 \times 10^{-6}$ \\
0.2 & $7.49625 \times 10^{-8}$ & $4.9975 \times 10^{-8}$ & $4.46806 \times 10^{-6}$ \\
0.3 & $1.12444 \times 10^{-7}$ & $7.49625 \times 10^{-8}$ & $6.70209 \times 10^{-6}$ \\
0.4 & $1.49925 \times 10^{-7}$ & $9.995 \times 10^{-8}$ & $8.93612 \times 10^{-6}$ \\
0.5 & $1.87406 \times 10^{-7}$ & $1.24937 \times 10^{-7}$ & $1.11702 \times 10^{-5}$ \\
0.6 & $2.24887 \times 10^{-7}$ & $1.49925 \times 10^{-7}$ & $1.34042 \times 10^{-5}$ \\
0.7 & $2.62369 \times 10^{-7}$ & $1.74912 \times 10^{-7}$ & $1.56382 \times 10^{-5}$ \\
0.8 & $2.9985 \times 10^{-7}$ & $1.999 \times 10^{-7}$ & $1.78722 \times 10^{-5}$ \\
0.9 & $3.37331 \times 10^{-7}$ & $2.24887 \times 10^{-7}$ & $2.01063 \times 10^{-5}$ \\
1.0 & $3.74812 \times 10^{-7}$ & $2.49875 \times 10^{-7}$ & $2.23403 \times 10^{-5}$ \\
\hline
\end{tabular}

TABLE 5: Comparison of absolute errors obtained by OHAM and ADM [5] for $\alpha=0.001, \beta=0.001$, and $\delta=1$.

\begin{tabular}{lcccccc}
\hline$t$ & ADM for & OHAM & ADM & OHAM & ADM & OHAM \\
& $x=0.1$ & $x=0.1$ & $x=0.5$ & $x=0.5$ & $x=0.9$ & $x=0.9$ \\
\hline 0.005 & $9.68763 \times 10^{-6}$ & $1.12257 \times 10^{-7}$ & $9.68691 \times 10^{-6}$ & $2.28888 \times 10^{-7}$ & $9.68619 \times 10^{-6}$ & $2.28888 \times 10^{-7}$ \\
0.001 & $1.93753 \times 10^{-6}$ & $2.24513 \times 10^{-8}$ & $1.93738 \times 10^{-6}$ & $4.57775 \times 10^{-8}$ & $1.93724 \times 10^{-6}$ & $4.57775 \times 10^{-8}$ \\
0.01 & $1.93752 \times 10^{-5}$ & $2.24514 \times 10^{-7}$ & $1.93738 \times 10^{-5}$ & $4.57777 \times 10^{-7}$ & $1.93724 \times 10^{-5}$ & $4.57777 \times 10^{-7}$ \\
\hline
\end{tabular}

First-Order Problem

$$
\begin{gathered}
\frac{\partial u_{1}(x, t)}{\partial t}-\left(1+C_{1}\right) \frac{\partial u_{0}(x, t)}{\partial t}-0.001 C_{1} u_{0}(x, t) \frac{\partial u_{0}(x, t)}{\partial x} \\
-0.001 C_{1} u_{0}(x, t)\left(1-u_{0}(x, t)\right)+C_{1} \frac{\partial^{2} u_{0}(x, t)}{\partial x^{2}}=0, \\
u_{1}(x, 0)=0 .
\end{gathered}
$$

Its solution is

$$
\begin{aligned}
& u_{1}\left(x, t, C_{1}\right) \\
& =-t\left(0.00025 C_{1}+6.25 \times 10^{-8} C_{1}\right. \\
& \quad \times \operatorname{sech}^{2}(0.00025 x) \\
& \left.\quad-0.00025 C_{1} \tanh ^{2}(0.00025 x)\right) .
\end{aligned}
$$

\section{Second-Order Problem}

$$
\begin{aligned}
& \frac{\partial u_{2}(x, t)}{\partial t}-\left(1+C_{1}\right) \frac{\partial u_{1}(x, t)}{\partial t}+0.001 C_{2} u_{0}(x, t) \\
& \times\left(1-u_{0}(x, t)\right)+C_{2} \frac{\partial^{2} u_{0}(x, t)}{\partial x^{2}} \\
& -C_{2} \frac{\partial u_{0}(x, t)}{\partial t}-0.001 C_{2} u_{0}(x, t) \frac{\partial u_{0}(x, t)}{\partial x} \\
& -0.001 C_{1} u_{1}(x, t) \frac{\partial u_{0}(x, t)}{\partial x}-0.001 C_{1} u_{0}(x, t)
\end{aligned}
$$

$$
\begin{gathered}
\times \frac{\partial u_{1}(x, t)}{\partial x}+C_{1} \frac{\partial^{2} u_{1}(x, t)}{\partial x^{2}}-0.001 C_{1} u_{1}(x, t) \\
-0.002 C_{1} u_{0}(x, t) u_{1}(x, t)=0, \\
u_{2}(x, 0)=0 .
\end{gathered}
$$

Its solution is

$$
\begin{aligned}
u_{2}\left(x, t, C_{1}, C_{2}\right) \\
=\operatorname{sech}^{5}(0.00025 x) \\
\times\left(-0.000187547 C_{1} t-0.000187547 C_{2} t\right. \\
\left.\quad+C_{1}^{2}(-0.000187547 t)\right) \cosh (0.00025 x) \\
+-0.0000625156 C_{1} t-0.0000625156 C_{2} t \\
+C_{1}^{2}(-0.0000625156 t)+2.71051 \\
\times 10^{-20} C_{1} t \sinh (0.00025 x)+2.71051 \\
\times 10^{-20} C_{1}^{2} t \sinh (0.00025 x)+2.71051 \\
\times 10^{-20} C_{2} t \sinh (0.00025 x)+3.12656 \\
\times 10^{-8} C_{1}^{2} t^{2} \sinh (0.00025 x)+3.12656 \\
\times 10^{-8} C_{1}^{2} t^{2} \sinh (0.00075 x)
\end{aligned}
$$


TABLE 6: Comparison of absolute errors obtained by OHAM and ADM [5] for $\alpha=1, \beta=1$, and $\delta=2$.

\begin{tabular}{lcccccc}
\hline$t$ & ADM for & OHAM & ADM & OHAM & ADM & OHAM \\
& $x=0.1$ & $x=0.1$ & $x=0.5$ & $x=0.5$ & $x=0.9$ & $x=0.9$ \\
\hline 0.0005 & $1.40177 \times 10^{-3}$ & $5.87633 \times 10^{-5}$ & $1.34526 \times 10^{-3}$ & $1.06736 \times 10^{-5}$ & $1.27699 \times 10^{-3}$ & $4.64718 \times 10^{-5}$ \\
0.0001 & $2.80396 \times 10^{-4}$ & $1.17539 \times 10^{-5}$ & $2.69094 \times 10^{-4}$ & $5.33686 \times 10^{-5}$ & $2.55438 \times 10^{-4}$ & $9.29303 \times 10^{-6}$ \\
0.001 & $2.80301 \times 10^{-3}$ & $1.17512 \times 10^{-4}$ & $2.69000 \times 10^{-3}$ & $1.06739 \times 10^{-4}$ & $2.55346 \times 10^{-3}$ & $9.296 \times 10^{-4}$ \\
\hline
\end{tabular}

TABLE 7: Absolute errors of OHAM for $x=2$ and $t \in[0.1,1]$.

\begin{tabular}{lcc}
\hline$t$ & $\alpha=0.001, \beta=0.001$, and & $\begin{array}{c}\alpha=0.001, \beta=0.001, \\
\text { and } \delta=2\end{array}$ \\
\hline 0.1 & $1.98526 \times 10^{-9}$ & $1.09926 \times 10^{-5}$ \\
0.2 & $3.20807 \times 10^{-8}$ & $2.19856 \times 10^{-5}$ \\
0.3 & $1.63084 \times 10^{-7}$ & $2.9789 \times 10^{-5}$ \\
0.4 & $5.16881 \times 10^{-7}$ & $4.39726 \times 10^{-5}$ \\
0.5 & $1.26475 \times 10^{-6}$ & $5.49666 \times 10^{-5}$ \\
0.6 & $2.62763 \times 10^{-6}$ & $6.5961 \times 10^{-5}$ \\
0.7 & $4.87621 \times 10^{-6}$ & $7.69557 \times 10^{-5}$ \\
0.8 & $8.33106 \times 10^{-6}$ & $8.79507 \times 10^{-5}$ \\
0.9 & $1.33626 \times 10^{-5}$ & $9.89461 \times 10^{-5}$ \\
\hline
\end{tabular}

The third order approximate solution using OHAM is given by

$$
\begin{aligned}
\tilde{u}\left(x, t, C_{1}, C_{2}\right)= & u_{0}(x, t)+u_{1}\left(x, t, C_{1}\right) \\
& +u_{2}\left(x, t, C_{1}, C_{2}\right)+u_{3}\left(x, t, C_{1}, C_{2}, C_{3}\right),
\end{aligned}
$$

where $u_{3}\left(x, t, C_{1}, C_{2}, C_{3}\right)$ is obtained in same lines as for first problem.

For the calculations of the constants $C_{1}, C_{2}$, and $C_{3}$ using the collocation method we have computed that

$$
\begin{gathered}
C_{1}=-5.928318703338053 \times 10^{-7}, \\
C_{2}=-465.9630543691778, \\
C_{3}=1.8651679832921486 .
\end{gathered}
$$

The third order OHAM solution yields very encouraging results after being compared with Fourth order approximate solution by ADM [5].

Table 5 shows a comparison between OHAM solution and $\mathrm{ADM}$ solution for $\alpha=0.001, \beta=0.001$, and $\delta=$ 1. Table 6 compares between OHAM solution and ADM solution for $\alpha=1, \beta=1$, and $\delta=2$. Table 7 shows the reliability of OHAM for larger domain.

\section{Conclusion}

We successfully applied OHAM for solution of Burger'sHuxley and Burger's-Fisher equations. The method is simple in applicability and is fast converging to the exact solution. The results obtained by OHAM are very consistent in comparison with ADM.

\section{References}

[1] C. A. J. Fletcher, "Burgers' equation: a model for all reasons," in Numerical Solutions of Partial Differential Equations (Parkville, 1981), J. Noye, Ed., pp. 139-225, North-Holland, Amsterdam, The Netherlands, 1982.

[2] D. L. Young, C. M. Fan, S. P. Hu, and S. N. Atluri, "The Eulerian-Lagrangian method of fundamental solutions for twodimensional unsteady Burgers' equations," Engineering Analysis with Boundary Elements, vol. 32, no. 5, pp. 395-412, 2008.

[3] J. M. Burgers, "Mathematical examples illustrating relations occurring in the theory of turbulent fluid motion," Transactions of the Royal Dutch Academy of Sciences in Amsterdam, vol. 17, no. 2, pp. 1-53, 1939.

[4] J. Satsuma, "Topics in soliton theory and exactly solvable nonlinear equations," in Proceedings of the Conference on Nonlinear Evolution Equations, Solitons and the Inverse Scattering Transform Held at the Mathematical Research Institute, Oberwolfach, July-August, 1986, M. Ablowitz, B. Fuchssteiner, and M. Kruskal, Eds., p. 342, World Scientific, Singapore, 1987.

[5] H. N. A. Ismail, K. Raslan, and A. A. Abd Rabboh, "Adomian decomposition method for Burger's-Huxley and Burger'sFisher equations," Applied Mathematics and Computation, vol. 159, no. 1, pp. 291-301, 2004.

[6] X. Y. Wang and Y. K. Lu, "Exact solutions of the extended Burgers-Fisher equation," Chinese Physics Letters, vol. 7, no. 4, pp. 145-147, 1990.

[7] M. Javidi, "Modified pseudospectral method for generalized Burger's-Fisher equation," International Mathematical Forum, vol. 1, no. 29-32, pp. 1555-1564, 2006.

[8] D. Kaya and S. M. El-Sayed, "A numerical simulation and explicit solutions of the generalized Burgers-Fisher equation," Applied Mathematics and Computation, vol. 152, no. 2, pp. 403413, 2004.

[9] P. Chandrasekaran and E. K. Ramasami, "Painleve analysis of a class of nonlinear diffusion equations," Journal of Applied Mathematics and Stochastic Analysis, vol. 9, no. 1, pp. 77-86, 1996.

[10] H. Chen and H. Zhang, "New multiple soliton solutions to the general Burgers-Fisher equation and the Kuramoto-Sivashinsky equation," Chaos, Solitons \& Fractals, vol. 19, no. 1, pp. 71-76, 2004.

[11] E. S. Fahmy, "Travelling wave solutions for some time-delayed equations through factorizations," Chaos, Solitons \& Fractals, vol. 38, no. 4, pp. 1209-1216, 2008.

[12] L. Jiang, Y.-C. Guo, and S.-J. Xu, "Some new exact solutions to the Burgers-Fisher equation and generalized Burgers-Fisher equation," Chinese Physics, vol. 16, no. 9, pp. 2514-2522, 2007.

[13] V. Marinca and N. Herişanu, "Application of optimal homotopy asymptotic method for solving nonlinear equations arising in heat transfer," International Communications in Heat and Mass Transfer, vol. 35, no. 6, pp. 710-715, 2008. 
[14] V. Marinca, N. Herişanu, C. Bota, and B. Marinca, "An optimal homotopy asymptotic method applied to the steady flow of a fourth-grade fluid past a porous plate," Applied Mathematics Letters, vol. 22, no. 2, pp. 245-251, 2009.

[15] V. Marinca, N. Herişanu, and I. Nemeş, "Optimal homotopy asymptotic method with application to thin film flow, Central European Journal of Physics, vol. 6, no. 3, pp. 648-653, 2008.

[16] R. Nawaz, M. N. Khalid, S. Islam, and S. Yasin, "Solution of tenth order boundary value problems using optimal homotopy asymptotic method (OHAM)," Canadian Journal on Computing in Mathematics, Natural Sciences, Engineering \& Medicin, vol. 1, no. 2, pp. 37-54, 2010.

[17] S. Iqbal, M. Idrees, A. M. Siddiqui, and A. R. Ansari, "Some solutions of the linear and nonlinear Klein-Gordon equations using the optimal homotopy asymptotic method," Applied Mathematics and Computation, vol. 216, no. 10, pp. 2898-2909, 2010.

[18] S. Haq, M. Idrees, and S. Isalam, "Application of optimal homotopy asymptotic Method to eighth order boundary value problems," Journal of Applied Mathematics and Computing, vol. 2, no. 4, pp. 38-47, 2008.

[19] M. Idrees, S. Haq, and S. Islam, "Application of optimal homotopy asymptotic method to fourth order boundary value problems," World Applied Sciences Journal, vol. 9, no. 2, pp. 131$137,2010$.

[20] M. Idrees, S. Islam, S. Haq, and S. Islam, "Application of the optimal homotopy asymptotic Method to squeezing flow," Computers and Mathematics with Applications, vol. 59, no. 12, pp. 3858-3866, 2010.

[21] M. Idrees, S. Haq, and S. Islam, "Application of optimal homotopy asymptotic method to special sixth order boundary value problems," World Applied Sciences Journal, vol. 9, no. 2, pp. 138-143, 2010.

[22] X. Y. Wang, Z. S. Zhu, and Y. K. Lu, "Solitary wave solutions of the generalised Burgers-Huxley equation," Journal of Physics A, vol. 23, no. 3, pp. 271-274, 1990. 


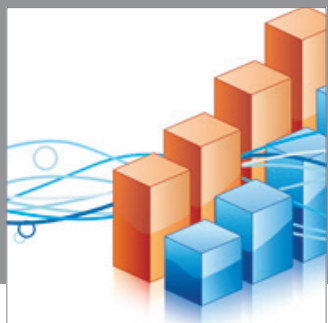

Advances in

Operations Research

mansans

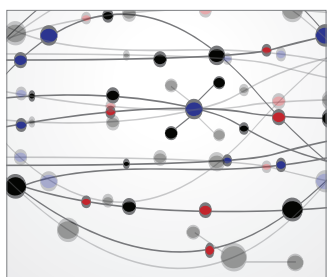

The Scientific World Journal
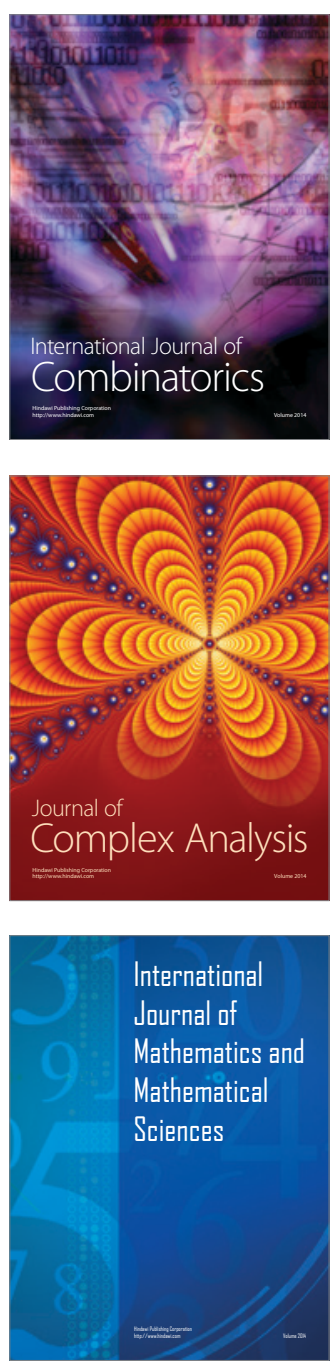
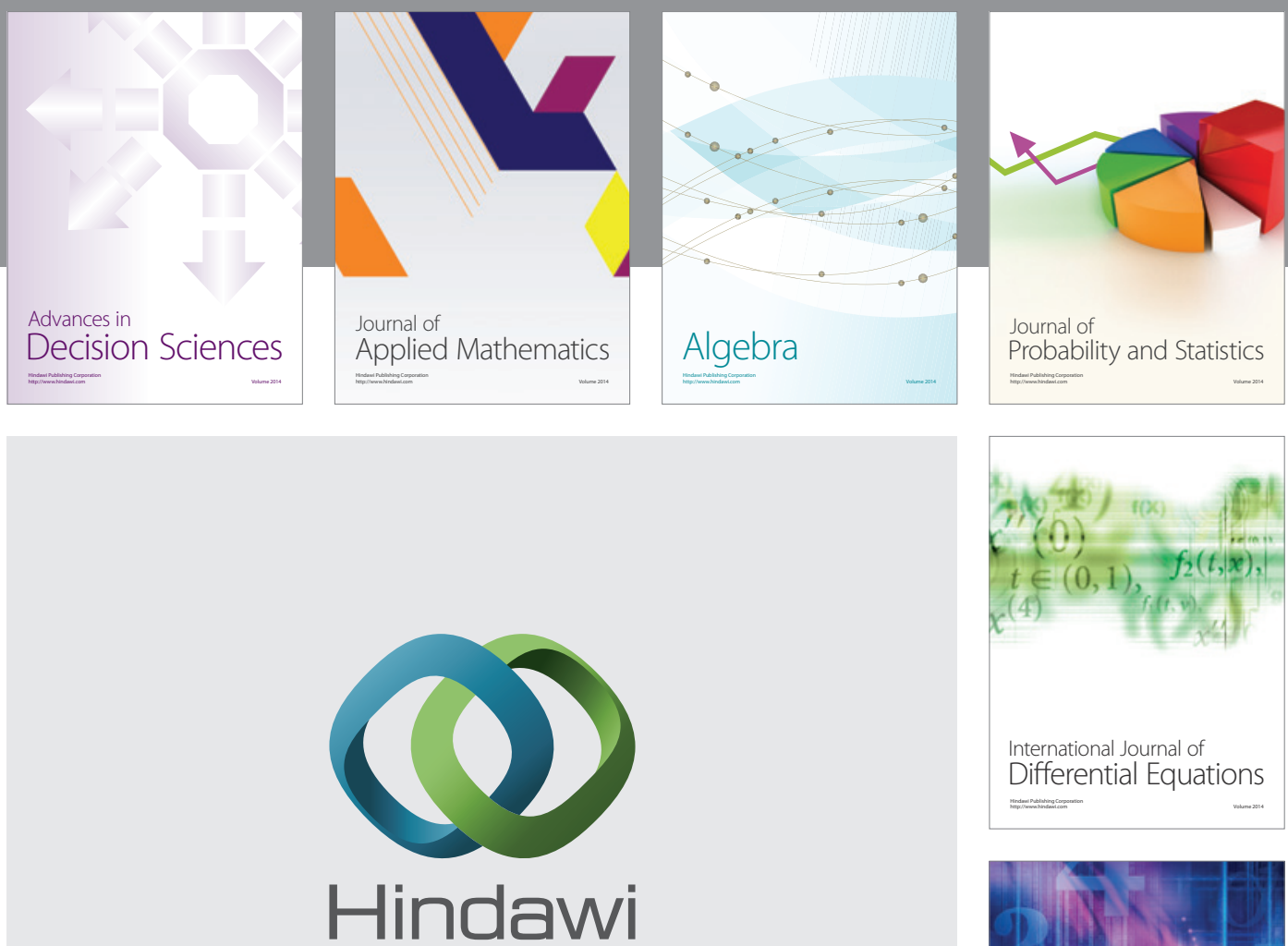

Submit your manuscripts at http://www.hindawi.com
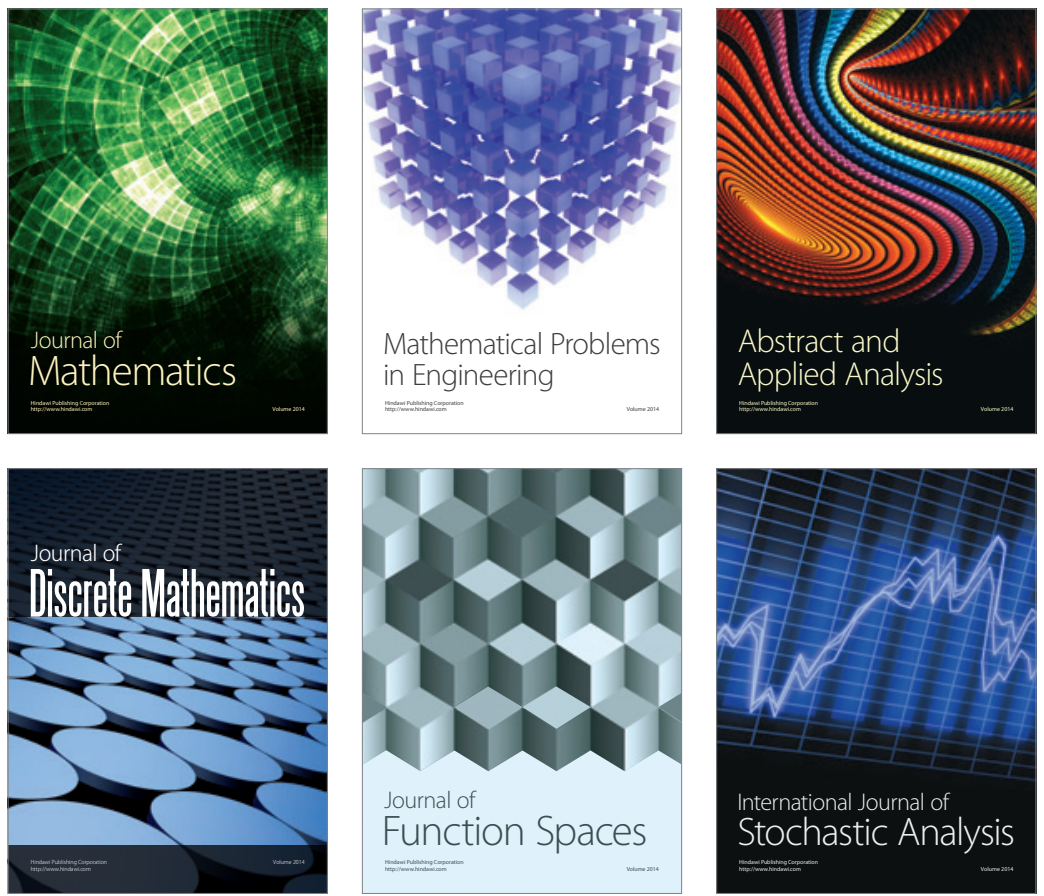

Journal of

Function Spaces

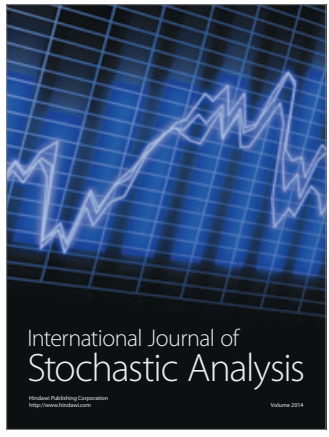

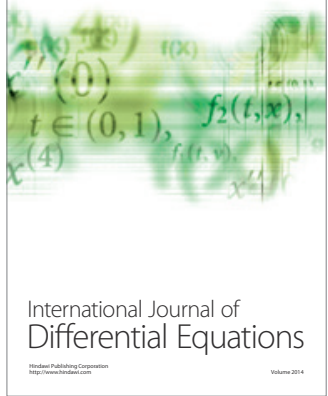
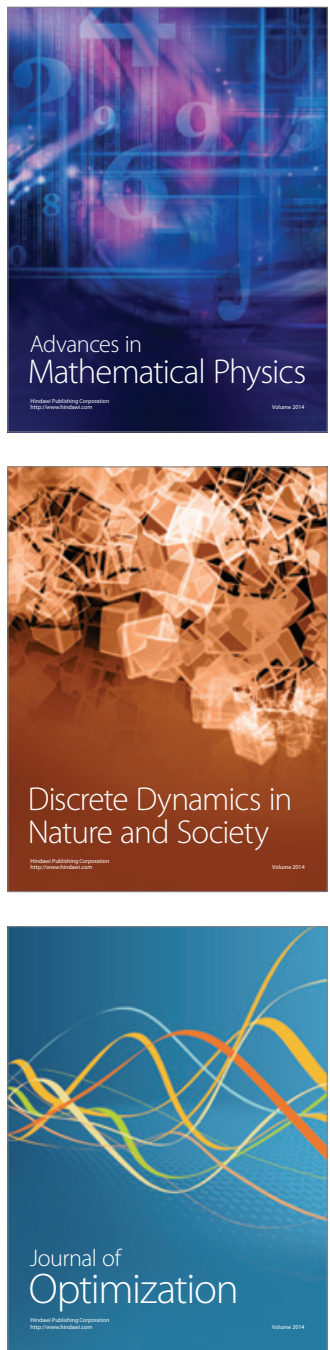